\title{
Temporal patterns of inflammatory biomarkers measured in the cerebrospinal fluid of patients with aneurysmal subarachnoid hemorrhage using multiplex Proximity Extension Assay technology.
}

Pavlos Vlachogiannis ( $\square$ pavlos.vlachogiannis@neuro.uu.se)

Uppsala Universitet https://orcid.org/0000-0002-8597-8862

Lars Hillered

Uppsala Universitet

Per Enblad

Uppsala Universitet

Elisabeth Ronne-Engström

Uppsala Universitet

\section{Research}

Keywords: subarachnoid hemorrhage, neuroinflammation, biomarkers, Proximity Extension Assay (PEA) technology

Posted Date: March 13th, 2020

DOI: https://doi.org/10.21203/rs.3.rs-17091/v1

License: (9) This work is licensed under a Creative Commons Attribution 4.0 International License.

Read Full License 


\section{Abstract}

Background Neuroinflammation has been extensively studied in the context of subarachnoid hemorrhage $(\mathrm{SAH})$ in recent years. A common approach is correlation of levels of single or few inflammatory biomarkers with clinical parameters such as cerebral vasospasm and outcome. However, the complexity of the inflammatory response post SAH may require a more comprehensive overall approach by analyzing multiple biomarkers simultaneously.

Methods Twenty-nine patients with SAH requiring insertion of external ventricular drainage were enrolled and ventricular cerebrospinal fluid was collected at days 1, 4 and 10 after hemorrhage. The levels of 92 inflammatory biomarkers were simultaneously measured in the samples using Proseek Multiplex Inflammation $1 \circledR$ assay (Olink Proteomics, Uppsala, Sweden) based on Proximity Extension Assay (PEA) technology. Thirty-eight proteins were excluded from further analysis due to lack of $>10 \%$ of measurable values. Temporal patterns for each of the remaining 54 proteins were illustrated on graphs with medians and quartiles. Wilcoxon matched pairs test was used for comparison of the medians between time points.

Results Four different patterns were visually observed with an early peak and gradually decreasing trend (9 proteins), middle peak (14 proteins), late peak after a gradually increasing trend (27 proteins) and no specific pattern (4 proteins). Several biomarkers showed statistically significant increasing trends (defined as day $1<$ day $4<$ day 10 values) and late peaks (day $4<$ day 10 ), such as chemokines CXCL6 and CCL23, or significant early peaks (that is, day $1>$ day 4) such as leukemia inhibitor factor (LIF) and macrophage inflammatory factor-1 $\beta$ (MIP-1 $\beta$ ). No statistically significant decreasing trends (defined as day $1>$ day $4>$ day 10 ) were observed. Two proteins showed statistically significant middle peaks (chemokine CCL28 and Delta and Notch epidermal growth factor-related receptor-DNER).

Conclusion The comprehensive data set provided in this study may act as an illustration of an inflammatory profile of the acute phase of SAH showing groups of biomarkers with similar temporal patterns of activation. Further studies can be designed based on these data in order to explore potential implications on early and late clinical events in the disease course.

\section{Background:}

Spontaneous subarachnoid hemorrhage (SAH) comprises approximately $5 \%$ of all strokes with an incidence of around 9 cases per 100.000 per year and aneurysm rupture being the cause in $85 \%$ of cases. [1] Brain injury in SAH occurs both at the time of the bleeding itself, termed primary injury, but also during the following days to weeks, a phenomenon known as secondary injury. Despite advances in the critical care of SAH patients and aneurysm treatment methods, mortality and morbidity rates among the survivals from the initial bleeding remain high, reflecting a lack of effective treatments targeting the pathophysiological mechanisms that underlie the secondary injury. 
Delayed cerebral ischemia ( $\mathrm{DCl}$ ) develops in approximately $30-40 \%$ of SAH patients and is considered to be a major cause of unfavorable outcome.[2] For decades the condition was attributed to cerebral vasospasm (CV), i.e. the narrowing of basal cerebral arteries seen early on angiography and persisting for up to two weeks post SAH leading to decreased cerebral blood flow (CBF) and infarctions in the affected territories.[3] However, about $20 \%$ of SAH patients develop DCI without evidence of CV and only $30 \%$ of patients with CV actually suffer from DCl.[4] Moreover, the randomized multicenter CONCSIOUS- 1 trial with the endothelin receptor antagonist Clazosentan failed to show any effect on functional outcome or incidence of cerebral ischemia despite significant decrease of CV.[5, 6] Thus, an uncoupling of angiographic vasospasm and $\mathrm{DCl}$ became apparent and new concepts emerged as potential underlying mechanisms of delayed brain injury.[7, 8]

Early brain injury (EBI) refers to the events occurring within the first $72 \mathrm{~h}$ from ictus and includes the primary injury and its direct consequences. Elevation of intracranial pressure (ICP), global ischemia, impairment of CBF autoregulation, cortical spreading depolarization (CSD), disruption of blood-brain barrier (BBB), cell death, oxidative stress and inflammatory processes are among the mechanisms that are activated shortly after aneurysm rupture and evolve during the following days.[9]

The processes triggered by the $\mathrm{EBI}$ are now believed to play an important role in the development of $\mathrm{DCl}$. $[9,10]$ Neuroinflammation may be a mechanistic link between these two conditions and has been studied extensively in the past years.[11-13] Many clinical and experimental studies report levels of single or few inflammatory biomarkers (such as cytokines) in the peripheral blood, cerebrospinal fluid (CSF) and cerebral extracellular fluid through microdialysis (MD) in SAH patients or animal models and further correlate these biomarkers with clinical parameters and outcome.[14-22] Although this strategy provides useful insight in the interplay between inflammation and the disease course it fails to account for the complexity of the involved mechanisms that cannot be adequately described by a single (or few) parameter(s) alone.

The aim of the present study was to provide a comprehensive inflammatory profile of the acute phase of SAH by simultaneously measuring the levels of 92 inflammatory biomarkers in the CSF at days 1, 4 and 10 after admission using multiplex Proximity Extension Assay (PEA) technology. The biomarkers were then categorized into different groups according to their temporal expression.

\section{Methods:}

\section{Ethics}

The study was conducted in accordance with Declaration of Helsinki for human studies and approved by Uppsala University Ethics Committee. All participants or their next of kin gave written consent for participation in the study.

\section{Patients}


Demographic data of the 29 patients included in the study are presented in Table 1. Inclusion period was between May 2013 and August 2014. Eligibility criteria was spontaneous SAH severe enough to require insertion of an external ventricular drain (EVD) within $24 \mathrm{~h}$ from ictus. Patients considered terminally ill from the bleeding were excluded. Brain CT scans were used to establish the diagnosis of SAH and were classified according to Fisher scale (median 4).[23] World Federation of Neurosurgical societies (WFNS) and Hunt \& Hess scores were noted on admission (medians 4 and 3, respectively).[24, 25] CT angiography and catheter angiography were performed in all cases and aneurysms were identified in 28 patients. In 26 patients the aneurysms were endovascularly secured and the remaining two were surgically clipped. The patients were treated at the Neurointensive Care Unit of Uppsala University Hospital for at least 10 days after the bleeding. Standardized treatment protocols described in a previous publication were applied.[26] Functional outcome was assessed by a research nurse at 1 year using Glasgow Outcome Scale.[27]

Table 1

Demographic data of the patient cohort.

\begin{tabular}{|ll|}
\hline Number of patients & $\mathbf{2 9}$ \\
\hline Male vs Female & 12 vs 17 \\
\hline Mean age (range) & 57 (37-81) \\
\hline Median Hunt \& Hess score & 3 \\
\hline Median World Federation of Neurosurgical Societies score & 4 \\
\hline Median Fisher grade & 4 \\
\hline Anterior vs posterior circulation aneurysm & 23 vs 5 (1 patient with no aneurysm) \\
\hline Embolization vs surgery & 26 vs 2 \\
\hline Favorable vs unfavorable outcome (Glasgow Outcome Scale) & 9 vs 20 \\
\hline CSF Samples and analySis & \\
\hline
\end{tabular}

Ventricular CSF samples were collected through the EVD within $24 \mathrm{~h}$, on day 4 and on day 9-11 after the bleeding, centrifuged directly after collection and frozen in $-70^{\circ} \mathrm{C}$. The samples were then analyzed using Proseek Multiplex Inflammation $1 \circledR$, a multiplex assay panel manufactured by Olink Proteomics AB, Uppsala, Sweden where 92 inflammation-related biomarkers are simultaneously measured using Proximity Extension Assay (PEA) technology (available online at https://www.olink.com/products/inflammation). The analytical method was previously described in detail.[28] Values are provided in output unit Normalized Protein Expression (NPX) on log2 scale. NPX values express relative quantification between samples but is not an absolute quantification. Limit of detection (LOD) is determined for each biomarker based on the negative controls analyzed in each run.

\section{Biomarkers}



A list of the 92 biomarkers included in the panel as well as their families is provided in Table 2. In summary, the panel included 31 cytokines, 20 chemokines, 9 growth factors, 8 tumor necrosis factor (TNF)-family members, 6 membrane glycoproteins, 6 neurotrophic factors, 5 proteases and 7 miscellaneous proteins. A total of 87 NPX values (29 patients $\times 3$ time points) corresponding to protein concentrations were collected for each biomarker. Variations in the detectability of the biomarkers in the samples were noticed; for example, in 44 proteins all samples were successfully analyzed while in 6 proteins all values were below limit of detection. An inclusion criterion was therefore defined that the values needed to be above level of detection (LOD) in at least $90 \%$ of the samples for each protein (that is $<9$ missing values), which lead to the exclusion of 38 proteins, thus leaving 54 proteins for further analysis. 
Table 2

List of biomarkers included in the panel

\begin{tabular}{|c|c|c|c|}
\hline $\mathrm{Nr}$ & PROTEIN & FULL NAME & FAMILY \\
\hline 1 & 4E-BP1 & $\begin{array}{l}\text { Eukaryotic translation initiation factor } 4 \text { E-binding } \\
\text { protein } 1\end{array}$ & Translation factor \\
\hline 2 & ADA & Adenosine deaminase & Deaminase \\
\hline 3 & ARTN & Artemin & Neurotrophic factor \\
\hline 4 & AXIN1 & Axin-1 & G-protein regulator \\
\hline 5 & BDNF & Brain-derived neurotrophic factor & Neurotrophic factor \\
\hline 6 & beta-NGF & Beta-nerve growth factor & Neurotrophic factor \\
\hline 7 & CASP-8 & Caspase-8 & Cysteine protease \\
\hline 8 & CCL11 & C-C motif chemokine ligand 11 (Eotaxin) & Chemokine \\
\hline 9 & $\begin{array}{l}\text { CCL13 (MCP- } \\
4)\end{array}$ & $\begin{array}{l}\text { C-C motif chemokine ligand } 13 \text { (monocyte } \\
\text { chemotactic protein 4) }\end{array}$ & Chemokine \\
\hline 10 & CCL19 & C-C motif chemokine ligand 19 & Chemokine \\
\hline 11 & CCL2 (MCP-1) & $\begin{array}{l}\text { C-C motif chemokine ligand } 2 \text { (monocyte } \\
\text { chemotactic protein } 1 \text { ) }\end{array}$ & Chemokine \\
\hline 12 & CCL20 & C-C motif chemokine ligand 20 & Chemokine \\
\hline 13 & CCL23 (MIP-3) & $\begin{array}{l}\text { C-C motif chemokine ligand } 23 \text { (macrophage } \\
\text { inflammatory protein-3) }\end{array}$ & Chemokine \\
\hline 14 & CCL25 & C-C motif chemokine ligand 25 & Chemokine \\
\hline 15 & CCL28 & C-C motif chemokine ligand 28 & Chemokine \\
\hline 16 & CCL3 (MIP-1a) & $\begin{array}{l}\text { C-C motif chemokine ligand } 3 \text { (macrophage } \\
\text { inflammatory protein-1a) }\end{array}$ & Chemokine \\
\hline 17 & CCL4 (MIP-1 $\beta)$ & $\begin{array}{l}\text { C-C motif chemokine ligand } 4 \text { (macrophage } \\
\text { inflammatory protein- } 1 \beta \text { ) }\end{array}$ & Chemokine \\
\hline 18 & CCL7 (MCP-3) & $\begin{array}{l}\text { C-C motif chemokine ligand } 7 \text { (monocyte } \\
\text { chemotactic protein } 3 \text { ) }\end{array}$ & Chemokine \\
\hline 19 & CCL8 (MCP-2) & $\begin{array}{l}\text { C-C motif chemokine ligand } 8 \text { (monocyte } \\
\text { chemotactic protein } 2 \text { ) }\end{array}$ & Chemokine \\
\hline
\end{tabular}

Alphabetic list of the 92 biomarkers included in Proseek Multiplex Inflammation $1 \circledast$ panel (abbreviations and full names) together with their families. The 54 biomarkers that met the criterion of being above level of detection in at least $90 \%$ of the samples (that is $<9$ missing values for each protein) to be included for further analysis are marked in bold. 


\begin{tabular}{|c|c|c|c|}
\hline $\mathrm{Nr}$ & PROTEIN & FULL NAME & FAMILY \\
\hline 20 & CD244 & Natural killer cell receptor 2B4 & $\begin{array}{l}\text { Membrane } \\
\text { glycoprotein }\end{array}$ \\
\hline 21 & CD40 & $\begin{array}{l}\text { Tumor necrosis factor receptor superfamily member } \\
5\end{array}$ & $\begin{array}{l}\text { TNF receptor } \\
\text { superfamily }\end{array}$ \\
\hline 22 & CD5 & T-cell surface glycoprotein CD5 & $\begin{array}{l}\text { Membrane } \\
\text { glycoprotein }\end{array}$ \\
\hline 23 & CD6 & T-cell differentiation antigen CD6 & $\begin{array}{l}\text { Membrane } \\
\text { glycoprotein }\end{array}$ \\
\hline 24 & CDCP-1 & CUB domain-containing protein 1 & $\begin{array}{l}\text { Membrane } \\
\text { glycoprotein }\end{array}$ \\
\hline 25 & CSF-1 & Macrophage colony-stimulating factor 1 & Cytokine \\
\hline 26 & CST5 & Cystatin-D & $\begin{array}{l}\text { Cysteine proteinase } \\
\text { inhibitor }\end{array}$ \\
\hline 27 & CX3CL1 & C-X3-C motif chemokine ligand 1 (Fractalkine) & Chemokine \\
\hline 28 & CXCL1 & C-X-C motif chemokine ligand 1 & Chemokine \\
\hline 29 & CXCL10 & C-X-C motif chemokine ligand 10 & Chemokine \\
\hline 30 & CXCL11 & C-X-C motif chemokine ligand 11 & Chemokine \\
\hline 31 & CXCL5 & C-X-C motif chemokine ligand 5 & Chemokine \\
\hline 32 & CXCL6 & C-X-C motif chemokine ligand 6 & Chemokine \\
\hline 33 & CXCL9 & C-X-C motif chemokine ligand 9 & Chemokine \\
\hline 34 & DNER & $\begin{array}{l}\text { Delta and Notch-like epidermal growth factor-related } \\
\text { receptor }\end{array}$ & Growth factor \\
\hline 35 & EN-RAGE & Protein S100-A12 & $\begin{array}{l}\text { Calcium-binding } \\
\text { protein }\end{array}$ \\
\hline 36 & FGF19 & Fibroblast growth factor 19 & Growth factor \\
\hline 37 & FGF-21 & Fibroblast growth factor 21 & Growth factor \\
\hline 38 & FGF-23 & Fibroblast growth factor 23 & Growth factor \\
\hline 39 & FGF-5 & Fibroblast growth factor 5 & Growth factor \\
\hline 40 & Flt3L & Fms-related tyrosine kinase 3 ligand & Cytokine \\
\hline
\end{tabular}

Alphabetic list of the 92 biomarkers included in Proseek Multiplex Inflammation $1{ }^{\circledR}$ panel (abbreviations and full names) together with their families. The 54 biomarkers that met the criterion of being above level of detection in at least $90 \%$ of the samples (that is $<9$ missing values for each protein) to be included for further analysis are marked in bold. 


\begin{tabular}{|c|c|c|c|}
\hline $\mathrm{Nr}$ & PROTEIN & FULL NAME & FAMILY \\
\hline 41 & GDNF & Glial cell line-derived neurotrophic factor & Neurotrophic factor \\
\hline 42 & HGF & Hepatocyte growth factor & Growth factor \\
\hline 43 & IFN-gamma & Interferon gamma & Cytokine \\
\hline 44 & IL-10 & Interleukin-10 & Cytokine \\
\hline 45 & IL-10RA & Interleukin-10 receptor subunit alpha & Cytokine \\
\hline 46 & IL-10RB & Interleukin-10 receptor subunit beta & Cytokine \\
\hline 47 & IL-12B & Interleukin-12 subunit beta & Cytokine \\
\hline 48 & IL-13 & Interleukin-13 & Cytokine \\
\hline 49 & IL-15RA & Interleukin-15 receptor subunit alpha & Cytokine \\
\hline 50 & IL-17A & Interleukin-17A & Cytokine \\
\hline 51 & IL-17C & Interleukin-17C & Cytokine \\
\hline 52 & IL-18 & Interleukin-18 & Cytokine \\
\hline 53 & IL-18R1 & Interleukin-18 receptor 1 & Cytokine \\
\hline 54 & IL-1a & Interleukin-1 alpha & Cytokine \\
\hline 55 & IL-2 & Interleukin-2 & Cytokine \\
\hline 56 & IL-2RB & Interleukin-2 receptor subunit beta & Cytokine \\
\hline 57 & IL-20 & Interleukin-20 & Cytokine \\
\hline 58 & IL-20RA & Interleukin-20 receptor subunit alpha & Cytokine \\
\hline 59 & IL-22 RA1 & Interleukin-20 receptor subunit alpha-1 & Cytokine \\
\hline 60 & IL-24 & Interleukin-24 & Cytokine \\
\hline 61 & IL-33 & Interleukin-33 & Cytokine \\
\hline 62 & IL-4 & Interleukin-4 & Cytokine \\
\hline 63 & IL-5 & Interleukin-5 & Cytokine \\
\hline 64 & IL-6 & Interleukin-6 & Cytokine \\
\hline 65 & IL-7 & Interleukin-7 & Cytokine \\
\hline
\end{tabular}

Alphabetic list of the 92 biomarkers included in Proseek Multiplex Inflammation $1 \AA$ panel (abbreviations and full names) together with their families. The 54 biomarkers that met the criterion of being above level of detection in at least $90 \%$ of the samples (that is $<9$ missing values for each protein) to be included for further analysis are marked in bold. 


\begin{tabular}{|c|c|c|c|}
\hline $\mathrm{Nr}$ & PROTEIN & FULL NAME & FAMILY \\
\hline 66 & IL-8 & Interleukin-8 & Cytokine \\
\hline 67 & LIF & Leukemia inhibitory factor & Cytokine \\
\hline 68 & LIF-R & Leukemia inhibitory factor receptor & Cytokine \\
\hline 69 & MMP-1 & Matrix metalloproteinase-1 & Protease \\
\hline 70 & MMP-10 & Matrix metalloproteinase-10 & Protease \\
\hline 71 & NRTN & Neurturin & Neurotrophic factor \\
\hline 72 & NT-3 & Neurotrophin-3 & Neurotrophic factor \\
\hline 73 & OPG & Osteoprotegerin & $\begin{array}{l}\text { TNF receptor } \\
\text { superfamily }\end{array}$ \\
\hline 74 & OSM & Oncostatin-M & Cytokine \\
\hline 75 & PD-L1 & Programmed cell death ligand 1 & $\begin{array}{l}\text { Membrane } \\
\text { glycoprotein }\end{array}$ \\
\hline 76 & SCF & Stem cell factor & Cytokine \\
\hline 77 & SIRT2 & SIR2-like protein 2 & $\begin{array}{l}\text { NAD dependent } \\
\text { deacetylase }\end{array}$ \\
\hline 78 & SLAMF1 & Signaling lymphocytic activation molecule & $\begin{array}{l}\text { Membrane } \\
\text { glycoprotein }\end{array}$ \\
\hline 79 & ST1A1 & Sulfotransferase $1 \mathrm{~A} 1$ & Transferase \\
\hline 80 & STAMPB & STAM-binding protein & Protease \\
\hline 81 & TGFA & Transforming growth factor alpha & Growth factor \\
\hline 82 & TGF- $\beta 1$ & Transforming growth factor beta- 1 & Growth factor \\
\hline 83 & TNF & Tumor necrosis factor & TNF family \\
\hline 84 & TNFB & Tumor necrosis factor beta (Lymphotoxin-alpha) & Cytokine \\
\hline 85 & TNFRSF9 & $\begin{array}{l}\text { Tumor necrosis factor receptor superfamily member } \\
9\end{array}$ & $\begin{array}{l}\text { TNF receptor } \\
\text { superfamily }\end{array}$ \\
\hline 86 & TNFSF14 & Tumor necrosis factor superfamily member 14 & $\begin{array}{l}\text { TNF receptor } \\
\text { superfamily }\end{array}$ \\
\hline 87 & $\begin{array}{l}\text { TRAIL } \\
\text { (TNFSF10) }\end{array}$ & $\begin{array}{l}\text { TNF-related apoptosis-inducing ligand (TNF- } \\
\text { superfamily member 10) }\end{array}$ & $\begin{array}{l}\text { TNF receptor } \\
\text { superfamily }\end{array}$ \\
\hline \multicolumn{4}{|c|}{$\begin{array}{l}\text { Alphabetic list of the } 92 \text { biomarkers included in Proseek Multiplex Inflammation } 1 \circledast \text { panel } \\
\text { (abbreviations and full names) together with their families. The } 54 \text { biomarkers that met the criterion } \\
\text { of being above level of detection in at least } 90 \% \text { of the samples (that is }<9 \text { missing values for each } \\
\text { protein) to be included for further analysis are marked in bold. }\end{array}$} \\
\hline
\end{tabular}




\begin{tabular}{|llll|}
\hline Nr & PROTEIN & FULL NAME & FAMILY \\
\hline 88 & $\begin{array}{l}\text { TRANCE } \\
\text { (TNFSF11) }\end{array}$ & Tumor necrosis factor superfamily member 11 & $\begin{array}{l}\text { TNF receptor } \\
\text { superfamily }\end{array}$ \\
\hline 89 & TSLP & Thymic stromal lymphopoietin & Cytokine \\
\hline 90 & $\begin{array}{l}\text { TWEAK } \\
\text { (TNFSF12) }\end{array}$ & Tumor necrosis factor superfamily member 12 & $\begin{array}{l}\text { TNF receptor } \\
\text { superfamily }\end{array}$ \\
\hline 91 & uPA & Urokinase-type plasminogen activator & Protease \\
\hline 92 & VEGF-A & Vascular endothelial growth factor A & Growth factor \\
\hline $\begin{array}{l}\text { Alphabetic list of the 92 biomarkers included in Proseek Multiplex Inflammation 1 } 8 \text { panel } \\
\text { (abbreviations and full names) together with their families. The } 54 \text { biomarkers that met the criterion } \\
\text { of being above level of detection in at least 90\% of the samples (that is }<9 \text { missing values for each } \\
\text { protein) to be included for further analysis are marked in bold. }\end{array}$ \\
\hline
\end{tabular}

\section{Statistics}

The NPX values of the remaining 54 proteins were converted into linear scale using the formula $2^{\mathrm{NPX}}=$ linear NPX in order to better visualize the trends of the biomarkers during the observation period. Median values for each day and protein were then calculated from the linear NPX values and their development over time was illustrated in graphs with upper and lower quartiles. Statistical analysis was done on the linear NPX data that were visually not normally distributed. Therefore non-parametric statistical methods were chosen. Wilcoxon matched pairs test was used for the comparison of day 1 vs day 4 vs day 10 median values for each protein. Protein expression levels in graphs were illustrated with medians and upper and lower quartiles. The results were considered statistically significant at the $p<0.05$ level. All statistical analyses and graphical presentations were performed using the Statistica ${ }^{\circledR}$ software (Stat Soft, Inc., Tulsa, OK, USA).

\section{Results:}

Visual inspection of the linear NPX graphs revealed four different temporal patterns (Table 3): an early peak at day 1 followed by a decreasing trend (Group A: 9 proteins), a middle peak at day 4 (Group B: 14 proteins), an increasing trend with a late peak at day 10 (Group C: 27 proteins) and finally no specific pattern (Group D: 4 proteins). Figures 1-4 illustrate typical graphs of median protein expression levels (linear NPX) and upper and lower quartiles over time for each group. Medians were then compared between time points for each protein using Wilcoxon matched pairs test. Table 4 shows the results ( $p$ value considered significant at $<0.05$ ). 
Table 3

Groups of biomarkers by their temporal pattern of protein expression

\begin{tabular}{|c|c|c|c|}
\hline GROUP A $(n=9)$ & GROUP B $(n=14)$ & GROUP C $(n=27)$ & GROUP D $(n=4)$ \\
\hline EARLY PEAK & MIDDLE PEAK & LATE PEAK & NO PATTERN \\
\hline CCL11 & CCL28 & 4E-BP1 & CCL25 \\
\hline CCL20 & CSF-1 & $\mathrm{ADA}$ & MCP-1 \\
\hline FGF-19 & CST-5 & CASP8 & TNFRSF9 \\
\hline LIF & DNER & CCL19 & VEGF-A \\
\hline MIP-1a (CCL3) & FGF-5 & CCL23 & \\
\hline MIP-1 $\beta$ (CCL4) & Flt3L & CD40 & \\
\hline MMP10 & IL-10 & CD 5 & \\
\hline TGFA & IL-10RB & CDCP1 & \\
\hline \multirow[t]{15}{*}{ TRAIL } & IL-18R1 & CX3CL1 & \\
\hline & IL-6 & CXCL1 & \\
\hline & LIF-R & CXCL10 & \\
\hline & MCP-3 & CXCL11 & \\
\hline & OSM & CXCL5 & \\
\hline & TGF- $\beta 1$ & CXCL6 & \\
\hline & & CXCL9 & \\
\hline & & EN-RAGE & \\
\hline & & HGF & \\
\hline & & IL-18 & \\
\hline & & IL-7 & \\
\hline & & IL-8 & \\
\hline & & MCP-2 & \\
\hline & & OPG & \\
\hline & & SCF & \\
\hline
\end{tabular}

Four different groups were identified based on visual inspection of the graphs, that is an early peak followed by a decreasing trend (Group A), a middle peak (Group B), a late peak after an increasing trend (Group C) and finally no specific pattern (Group D). 


\begin{tabular}{|c|c|c|c|}
\hline GROUP A $(n=9)$ & GROUP B $(n=14)$ & GROUP C $(n=27)$ & GROUP D $(n=4)$ \\
\hline EARLY PEAK & MIDDLE PEAK & LATE PEAK & NO PATTERN \\
\hline \multicolumn{4}{|c|}{ SIRT2 } \\
\hline \multicolumn{4}{|c|}{ STAMPB } \\
\hline \multicolumn{4}{|c|}{ TWEAK } \\
\hline \multicolumn{4}{|c|}{ UPA } \\
\hline $\begin{array}{l}\text { Four different gro } \\
\text { followed by a dec } \\
\text { trend (Group C) a }\end{array}$ & $\begin{array}{l}\text { re identified based } \\
\text { g trend (Group A), a } \\
\text { lly no specific patter }\end{array}$ & $\begin{array}{l}\text { al inspection of the } \\
\text { peak (Group B), a la } \\
\text { lp D). }\end{array}$ & $\begin{array}{l}\text { that is an early peak } \\
\text { after an increasing }\end{array}$ \\
\hline
\end{tabular}


Table 4

Expression levels of all proteins and time points.

\begin{tabular}{|c|c|c|c|c|c|c|}
\hline GROUP A & DAY 1 & $\begin{array}{l}1 \text { VS } \\
4\end{array}$ & DAY 4 & $\begin{array}{l}4 \text { VS } \\
10\end{array}$ & DAY 10 & $\begin{array}{l}1 \text { VS } \\
10\end{array}$ \\
\hline CCL11 & $8,5(5,2-12)$ & $\begin{array}{l}<, 01 \\
0,01\end{array}$ & $5,2(3,4-7,8)$ & 0,16 & $5,1(4,3-7,2)$ & $<0,01$ \\
\hline CCL20 & $57(17-176)$ & 0,32 & $51(23-108)$ & 0,08 & $43(27-97)$ & 0,25 \\
\hline FGF-19 & $17(12-21)$ & 0,07 & $16(11-20)$ & 0,62 & $13(9,2-21)$ & 0,16 \\
\hline LIF & $52(21-148)$ & $\begin{array}{l}<, 01 \\
0,01\end{array}$ & $13(7,8-22)$ & 0,93 & $14(6,1-34)$ & $<0,01$ \\
\hline $\begin{array}{l}\text { MIP-1a } \\
\text { (CCL3) }\end{array}$ & $9,6(5,2-25)$ & 0,13 & $8(5,1-11)$ & 0,99 & $7,7(6,6-12)$ & 0,17 \\
\hline $\begin{array}{l}\text { MIP-1 } \beta \\
\text { (CCL4) }\end{array}$ & $80(47-404)$ & $\begin{array}{l}<, 01 \\
0,01\end{array}$ & $36(22-64)$ & 0,44 & $35(28-59)$ & $<0,01$ \\
\hline MMP-10 & $17(9,9-35)$ & 0,20 & $14(12-19)$ & 0,06 & $12(8,8-18)$ & 0,01 \\
\hline TGFA & $7,7(5,7-11)$ & 0,23 & $6,9(5,4-8,5)$ & 0,03 & $6,5(5,3-7,3)$ & 0,01 \\
\hline TRAIL & $9,4(6,8-16)$ & 0,01 & $7,3(5,2-8,9)$ & 0,99 & $6,7(5,3-9,6)$ & 0,04 \\
\hline GROUP B & DAY 1 & $\begin{array}{l}1 \mathrm{VS} \\
4\end{array}$ & DAY 4 & $\begin{array}{l}4 \mathrm{VS} \\
10\end{array}$ & DAY 10 & $\begin{array}{l}1 \mathrm{VS} \\
10\end{array}$ \\
\hline CCL28 & $1,3(1,2-1,5)$ & $\begin{array}{l}<, 01 \\
0,01\end{array}$ & $1,6(1,5-1,7)$ & 0,02 & $1,5(1,3-1,6)$ & 0,07 \\
\hline CSF-1 & $87(48-126)$ & 0,18 & $102(74-131)$ & 0,06 & $80(50-111)$ & 0,61 \\
\hline CST5 & $45(38-61)$ & $\begin{array}{l}<, 01 \\
0,0\end{array}$ & $60(54-65)$ & 0,20 & $57(48-65)$ & 0,02 \\
\hline DNER & $138(112-216)$ & $\begin{array}{l}<, 01 \\
0,0\end{array}$ & $327(250-381)$ & 0,01 & $259(170-360)$ & $<0,01$ \\
\hline FGF-5 & $3,2(2,5-4,9)$ & $<$ & $6,7(5,5-11)$ & 0,15 & $6(4,2-9,7)$ & 0,01 \\
\hline Flt3L & $156(129-231)$ & $\dot{0}, 01$ & $267(185-374)$ & 0,82 & $285(166-367)$ & $<0,01$ \\
\hline IL-10 & $5,1(3,6-8)$ & 0,31 & $6,3(4,5-7,9)$ & 0,31 & $4,9(3,3-9,5)$ & 0,92 \\
\hline IL-10RB & $3,4(2,9-6,2)$ & $<$ & $7,1(4,7-9,6)$ & 0,26 & $7,9(4,3-12)$ & $<0,01$ \\
\hline
\end{tabular}




\begin{tabular}{|c|c|c|c|c|c|c|}
\hline GROUP A & DAY 1 & $\begin{array}{l}1 \text { VS } \\
4\end{array}$ & DAY 4 & $\begin{array}{l}4 \text { VS } \\
10\end{array}$ & DAY 10 & $\begin{array}{l}1 \text { VS } \\
10\end{array}$ \\
\hline IL-18R1 & $3,9(2,3-6,9)$ & 0,12 & $5,7(3,3-7,4)$ & 0,34 & $5,3(2,8-7,5)$ & 0,34 \\
\hline IL-6 & $\begin{array}{l}927(267- \\
2037)\end{array}$ & $\begin{array}{l}< \\
0,01\end{array}$ & $\begin{array}{l}2715(537- \\
5589)\end{array}$ & 0,64 & $\begin{array}{l}2119(611- \\
6838)\end{array}$ & $<0,01$ \\
\hline LIF-R & $4(3,3-5,6)$ & $\begin{array}{l}<, 01 \\
0,01\end{array}$ & $5,2(4,4-7,4)$ & 0,26 & $5(4,4-6,4)$ & 0,03 \\
\hline MCP-3 & $38(15-78)$ & 0,02 & $95(29-172)$ & 0,31 & $98(31-290)$ & $<0,01$ \\
\hline OSM & $11(8,1-30)$ & 0,02 & $30(9,4-53)$ & 0,41 & $31(13-61)$ & 0,03 \\
\hline TGF- $\beta 1$ & $13(9-25)$ & $\begin{array}{l}<, 01 \\
0,01\end{array}$ & $30(19-36)$ & 0,15 & $29(17-54)$ & $<0,01$ \\
\hline GROUP C & DAY 1 & $\begin{array}{l}1 \text { VS } \\
4\end{array}$ & DAY 4 & $\begin{array}{l}4 \mathrm{VS} \\
10\end{array}$ & DAY 10 & $\begin{array}{l}1 \mathrm{VS} \\
10\end{array}$ \\
\hline 4E-BP1 & $2,3(1,7-3,3)$ & $\begin{array}{l}<, 01 \\
0,01\end{array}$ & $21(3,5-71)$ & $\begin{array}{l}<, 01 \\
0,01\end{array}$ & $111(21-102)$ & $<0,01$ \\
\hline ADA & $16(10-23)$ & ${ }_{0,01}^{<}$ & $32(22-64)$ & 0,055 & $53(29-107)$ & $<0,01$ \\
\hline CASP-8 & $2,9(1,7-3,6)$ & 0,26 & $2,8(2,2-3,9)$ & $\begin{array}{l}<, 01 \\
0,01\end{array}$ & $5,6(2,9-11)$ & $<0,01$ \\
\hline CCL19 & $177(115-388)$ & $\begin{array}{l}< \\
0,01\end{array}$ & $\begin{array}{l}1772(748- \\
3642)\end{array}$ & 0,01 & $\begin{array}{l}2875(1196- \\
5501)\end{array}$ & $<0,01$ \\
\hline CCL23 & $12(7,1-25)$ & $\begin{array}{l}<, 01 \\
0,01\end{array}$ & $63(31-104)$ & $\begin{array}{l}<, 01 \\
0,01\end{array}$ & $207(83-331)$ & $<0,01$ \\
\hline CD40 & $64(43-101)$ & $\begin{array}{l}<, 01 \\
0,01\end{array}$ & 109 (75-189) & $\begin{array}{l}<, 01 \\
0,01\end{array}$ & $250(137-395)$ & $<0,01$ \\
\hline CD5 & $2(1,5-3,5)$ & 0,055 & $3,2(2,2-4,2)$ & 0,23 & $4(2,2-5,3)$ & 0,01 \\
\hline CDCP1 & $13(9-15)$ & $\begin{array}{l}<, 01 \\
0,01\end{array}$ & $17(15-26)$ & $\begin{array}{l}<, 01 \\
0,01\end{array}$ & $28(17-42)$ & $<0,01$ \\
\hline CX3CL1 & $6,5(5,7-8,1)$ & 0,10 & $9,5(6,7-11)$ & 0,19 & $11,7(6,9-17)$ & 0,07 \\
\hline CXCL1 & $183(82-356)$ & $\begin{array}{l}< \\
0,01\end{array}$ & $830(127-1066)$ & $\begin{array}{l}< \\
0,01\end{array}$ & $\begin{array}{l}1037 \text { (437- } \\
1973)\end{array}$ & $<0,01$ \\
\hline CXCL10 & $179(76-417)$ & $\begin{array}{l}<, 01 \\
0,01\end{array}$ & $\begin{array}{l}1573(1024- \\
4301)\end{array}$ & 0,25 & $\begin{array}{l}2530(1181- \\
3539)\end{array}$ & $<0,01$ \\
\hline
\end{tabular}

Comparison of median protein expression levels (linear NPX) with upper and lower quartiles within parentheses between time points for each protein (classified in groups according to Table 3);

Wilcoxon matched pairs test was used for comparison; $p$-values were considered significant at $<0.05$ level. 


\begin{tabular}{|c|c|c|c|c|c|c|}
\hline GROUP A & DAY 1 & $\begin{array}{l}1 \text { VS } \\
4\end{array}$ & DAY 4 & $\begin{array}{l}4 \text { VS } \\
10\end{array}$ & DAY 10 & $\begin{array}{l}1 \text { VS } \\
10\end{array}$ \\
\hline CXCL11 & $5,7(3-8)$ & $\begin{array}{l}<, 01 \\
0,01\end{array}$ & $11,8(8-22)$ & 0,68 & $19,5(10-39)$ & $<0,01$ \\
\hline CXCL5 & $252(144-572)$ & 0,68 & $271(160-753)$ & 0,73 & $437(185-737)$ & 0,56 \\
\hline CXCL6 & $21(8,8-44)$ & $\begin{array}{l}< \\
0,01\end{array}$ & $204(22-408)$ & $\hat{0}_{0,01}$ & $642(172-959)$ & $<0,01$ \\
\hline CXCL9 & $7,1(6,2-19)$ & $\begin{array}{l}<, 01 \\
0,01\end{array}$ & $25,9(15-42)$ & $\begin{array}{l}< \\
0,01\end{array}$ & $59(18-116)$ & $<0,01$ \\
\hline EN-RAGE & $2,9(2,5-4,2)$ & $\begin{array}{l}<, 01 \\
0,01\end{array}$ & $9,8(5,8-29)$ & 0,11 & $16(5,9-39)$ & $<0,01$ \\
\hline HGF & $36(27-70)$ & $\begin{array}{l}< \\
0,01\end{array}$ & $86(67-116)$ & 0,08 & $114(64-188)$ & $<0,01$ \\
\hline IL-18 & $6,3(4,3-10)$ & 0,33 & $9,8(3,5-20)$ & $\begin{array}{l}< \\
0,01\end{array}$ & $24(8,3-37)$ & $<0,01$ \\
\hline IL-7 & $2,3(2-2,5)$ & 0,01 & $2,4(2,2-3,4)$ & 0,31 & $2,8(2,5-3,4)$ & $<0,01$ \\
\hline IL-8 & $\begin{array}{l}6671(4906- \\
12663)\end{array}$ & 0,29 & $\begin{array}{l}10867(3084- \\
16185)\end{array}$ & 0,03 & $\begin{array}{l}15540(6006- \\
21024)\end{array}$ & 0,06 \\
\hline МСР-2 & $107(53-188)$ & $\begin{array}{l}<, 01 \\
0,01\end{array}$ & $404(126-644)$ & 0,01 & $527(198-1082)$ & $<0,01$ \\
\hline OPG & $\begin{array}{l}638(356- \\
1629)\end{array}$ & 0,24 & $\begin{array}{l}1076(646- \\
1584)\end{array}$ & 0,03 & $\begin{array}{l}1381(741- \\
2481)\end{array}$ & 0,03 \\
\hline SCF & $7,2(5,2-11)$ & 0,19 & $12(7,9-15)$ & 0,39 & $13(6,1-19)$ & 0,13 \\
\hline SIRT2 & $4,7(3,3-8,2)$ & 0,08 & $6,3(3,9-10)$ & 0,06 & $10(5,2-22)$ & $<0,01$ \\
\hline STAMPB & $3,6(2,4-4,2)$ & 0,40 & $3,5(2,9-6)$ & 0,04 & $6,3(3,2-8,9)$ & 0,01 \\
\hline TWEAK & $179(123-246)$ & $\begin{array}{l}<, 01 \\
0,01\end{array}$ & $271(202-438)$ & 0,16 & $326(214-497)$ & $<0,01$ \\
\hline uPA & $156(120-227)$ & $\begin{array}{l}<, 01 \\
0,01\end{array}$ & $385(244-565)$ & $\begin{array}{l}< \\
0,01\end{array}$ & $\begin{array}{l}1230(525- \\
2239)\end{array}$ & $<0,01$ \\
\hline GROUP D & DAY 1 & $\begin{array}{l}1 \mathrm{VS} \\
4\end{array}$ & DAY 4 & $\begin{array}{l}4 \mathrm{VS} \\
10\end{array}$ & DAY 10 & $\begin{array}{l}1 \mathrm{VS} \\
10\end{array}$ \\
\hline CCL25 & $2,5(2,2-3,6)$ & 0,04 & $2,2(2-2,5)$ & 0,04 & $2,5(2-3,9)$ & 0,58 \\
\hline
\end{tabular}

Comparison of median protein expression levels (linear NPX) with upper and lower quartiles within parentheses between time points for each protein (classified in groups according to Table 3); Wilcoxon matched pairs test was used for comparison; $p$-values were considered significant at $<0.05$ level. 


\begin{tabular}{|c|c|c|c|c|c|c|}
\hline GROUP A & DAY 1 & $\begin{array}{l}1 \text { VS } \\
4\end{array}$ & DAY 4 & $\begin{array}{l}4 \text { VS } \\
10\end{array}$ & DAY 10 & $\begin{array}{l}1 \text { VS } \\
10\end{array}$ \\
\hline MCP-1 & $\begin{array}{l}9428(7822- \\
10942)\end{array}$ & 0,50 & $\begin{array}{l}9491(7628- \\
10487)\end{array}$ & 0,85 & $\begin{array}{l}8998(7870- \\
10266)\end{array}$ & 0,18 \\
\hline TNFRSF9 & $7(3,5-10)$ & 0,17 & $7,2(5-9,8)$ & 0,96 & $8,1(3,7-11)$ & 0,31 \\
\hline VEGF-A & $\begin{array}{l}1039(520- \\
1414)\end{array}$ & 0,04 & $689(493-1007)$ & 0,10 & $996(535-1247)$ & 0,38 \\
\hline \multicolumn{7}{|c|}{$\begin{array}{l}\text { Comparison of median protein expression levels (linear NPX) with upper and lower quartiles within } \\
\text { parentheses between time points for each protein (classified in groups according to Table } 3 \text { ); } \\
\text { Wilcoxon matched pairs test was used for comparison; } p \text {-values were considered significant at }<0.05 \\
\text { level. }\end{array}$} \\
\hline
\end{tabular}

Group A biomarkers with statistically significant early peak, defined as significantly higher day 1 vs day 4 values, were CCL11, LIF, MIP-1 $\beta$ (CCL4) and TRAIL; no biomarkers in this group showed statistically significant decreasing trend (that is, significant differences between day 1 vs day 4 and day 4 vs day 10 values).

Several Group B biomarkers (Table 4) showed significantly higher values between day 4 vs day 1 (i.e. CCL28, CST5, DNER, FGF-5, FIt3L, IL-10RB, IL-6, LIF-R, MCP-3, OSM and TGF- $\beta 1$ ). Half of these biomarkers showed decreasing NPX values towards day 10 and the rest remained stable. The only biomarkers with statistically significant middle peak (that is, significant difference between day 4 vs day 1 and day 4 vs day 10 values) were CCL28 and DNER.

Fifteen of the 27 biomarkers in Group C (Table 4) showed statistically significant late peak defined as significantly higher day 10 vs day 4 values (4E-BP1, CASP-8, CCL19, CCL23, CD40, CDCP1, CXCL1, CXCL6, CXCL9, IL-18, IL-8, MCP-2, OPG, STAMPB and UPA). Ten of them also showed statistically significant increasing trend during the entire observation period, that is significantly higher day 10 vs day 4 and day 4 vs day 1 values (i.e. 4E-BP1, CCL19, CCL23, CD40, CDCP1, CXCL1, CXCL6, CXCL9, MCP-2 and uPA).

\section{Discussion:}

In this study we present a concise data set of the sequential production of multiple biomarkers of inflammation in the CSF of patients during the first 10 days post SAH. To our knowledge, this is the largest data set to describe the inflammatory profile of SAH ever reported with 92 inflammatory biomarkers included, many of which never studied in SAH patients previously. This is also the first report of Proximity Extension Assay (PEA) technology being used for measuring biomarker expression levels in the context of SAH. Similar studies were recently published in patients with traumatic brain injury (TBI) and trigeminal neuralgia, as well as numerous other non-neurosurgical conditions (such as neuropathic pain, cardiovascular diseases, gastric cancer, etc.).[29-31] 
The analyses in the present study were performed in CSF compartment alone (not plasma or cerebral interstitial fluid) as this seems more suitable to describe the disease pathophysiology, given also that major early and late clinical complications (i.e. CV and chronic hydrocephalus) spatially correlate best with this compartment. Many of the included proteins have previously been associated to SAH inflammation, for example IL-1 ra, IL-6, IL-8, TNF-a, LIF, MCP-1, and VEGF-A.[32] On the other hand, there is scarce or non-existing literature on many other proteins, some of which showed interesting temporal patterns and statistically significant peaks and trends, such as LIF, CCL11, CCL28, 4E-BP1, CD40, CXCL6, CXCL9, and IL-18.[20, 33-36]

\section{Group A}

Nine biomarkers showed higher day 1 values and decreasing trends throughout the observation period (Tables 3 and 4). Four of them (CCL11, LIF, MIP-1 $\beta$ and TRAIL) showed statistically significant early peaks with day $1>$ day 4 levels but no statistically significant decreasing trends were observed. MIP-1 $\beta$ (also known as CCL4) and CCL11 are members of the chemokine family (C-C motif) and are involved in chemotaxis of macrophages and activated T-cells, respectively, as well as other proinflammatory actions. Their early peak can possibly be associated to the recruitment of leukocytes at the site of the bleeding. LIF is a cytokine involved in activation of signaling pathways that regulate cell growth among other actions. A similar early peak in serum has been observed previously.[37] TRAIL, a member of the TNF superfamily (also known as TNFSF10) involved in apoptosis, has not been studied in the SAH literature.

\section{Group B}

Among the 14 proteins included in this group only two (CCL28 and DNER) showed statistically significant middle peaks, that is significantly higher day 4 values than both day 1 and day 10 . Production of the chemokine CCL28 is induced by other proinflammatory cytokines and its chemotactic actions are exerted on B and T cells and eosinophils. Delta and Notch-like Epidermal growth factor-Receptor (DNER) is an activator of NOTCH1 pathway. None of these biomarkers have been studied in a SAH context earlier and their potential involvement in the SAH complications, mainly CV that coincides temporally with the observed middle peaks, should be examined.

It should be noted that the majority of the biomarkers in this group showed a significant increase in their levels between day 1 and day $4(11 / 14$, Table 4$)$. In almost all cases though, with the exception of the two biomarkers named above, these dynamics seemed to wear off between day 4 and day 10. Interestingly, IL- 6 was recently studied by our group using a quantitative routine monoclonal antibody-based method in CSF samples from 44 patients with severe SAH. The results showed a very similar temporal IL-6 pattern as in the present study, with increasing values between day 1 and day 4 followed by decreasing values towards day 10 , although remaining higher than day 1 values.[38]

\section{Group C}

Half of the studied biomarkers $(n=27)$ showed higher levels towards the end of the observation period, reflecting a more prolonged activation post SAH that may indicate an involvement in the healing 
processes or the development of late complications, such as late vasospasm, posthemorrhagic hydrocephalus, etc. Fifteen biomarkers (4E-BP1, CASP-8, CCL19, CCL23, DC40, CDCP1, CXCL1, CXCL6, CXCL9, IL18, IL8, MCP-2, OPG, STAMPB and UPA) showed statistically significant late peaks, meaning significantly higher day 10 than day 4 values. Apart from CASP-8, IL-18, IL-8, OPG and STAMPB all the remaining proteins in the list showed statistically significant increasing trends throughout the observation period with day $1<$ day $4<$ day 10 values.

Chemokines CCL23, CXCL6 and CXCL9, all potent chemotactic agents for resting T-cells/monocytes, neutrophilic granulocytes and T-cells respectively, have not been studied in SAH patients earlier. Signaling pathway molecule 4E-BP1 has been implicated in the development of vasospasm in a canine SAH-model but no reports on human studied are available.[35] Protein CD40, a member of TNF family, is found on antigen-presenting cells and mediates multiple inflammatory responses. Elevated serum levels of CD40 have been associated with poor outcome and severity of neurological deficits in SAH patients.[36, 39] IL18 , a strong proinflammatory cytokine involved in the synthesis of inflammatory mediators, has recently been shown to be a predictor of early brain injury and clinical prognosis in SAH patients as elevated concentrations correlated to cerebral edema and acute hydrocephalus.[20] It should be noted that the observed temporal pattern of IL-18 in that study differed from our study as we demonstrated a late peak of this cytokine. Urokinase (or UPA) is a serum protease that activates plasminogen to plasmin which in turn leads to thrombolysis and tissue degradation. Plasma concentrations of its receptor (soluble uPAreceptor) was not shown to correlate with neurological outcome post SAH.[40]

\section{General considerations}

The great variability of the expression levels and temporal patterns of the measured biomarkers is an indicator of the complexity of the inflammatory response after SAH. Many of the included proteins are well established in the SAH research both in humans and in preclinical animal models while others are novel in the SAH field. Their exact role as well as interplay with each other is not easy to establish, especially considering the fact that many of these substances are described to play both a detrimental and a beneficial role in the disease course depending on the time after bleeding.[41] This study may offer guidance for further research on groups of biomarkers based either on their families or their pattern of activation (e.g. early vs late peak) to identify potential underlying inflammatory mechanisms of SAH complications as well as novel targets of intervention in order to prevent/treat these conditions and improve outcome.

\section{Limitations}

A limitation with the study is that the PEA analysis method in its presently available form does not give absolute protein concentrations. However, the protein expression levels illustrate the relative concentrations and how these change over time. They also illustrate the relations between the levels of the different biomarkers. The study may be limited by the relatively small number of patients included. Another drawback may be the fact that the study is limited to the CSF compartment. Similar analyses could be performed in the CSF, cerebral interstitial fluid and plasma, giving the opportunity to compare 
protein levels in the different fluid compartments providing a more thorough inflammatory profile of the acute phase of the disease. Comparisons with healthy individuals could also serve as an indicator of the intensity of activation for each biomarker. Moreover, correlations of the biomarker expression levels with clinical parameters were not performed in this study, as the main goal was to provide general information of as many proteins as possible in order to look for patterns of expression for further investigation. Finally, measurements in more than three time points could possibly describe the temporal patterns of each protein more accurately.

\section{Conclusion:}

The temporal patterns of expression of multiple inflammation-related proteins in the acute phase of SAH are reported in this study providing an inflammatory profile of the disease that facilitates further research in the field of biomarkers. Proximity Extension Assay technology enables the measurement of the expression levels of several biomarkers simultaneously in small amounts of sample, adding a useful tool in the quest of finding relevant biomarkers to better describe and understand the complex pathophysiology of SAH.

\section{Abbreviations}

SAH

subarachnoid haemorrhage

PEA

Proximity Extension Assay

$\mathrm{DCl}$

delayed cerebral ischemia

CV

cerebral vasospasm

CBF

cerebral blood flow

EBI

early brain injury

ICP

intracranial pressure

CSD

cortical spreading depolarization

BBB

blood-brain barrier

CSF

cerebrospinal fluid

MD 
microdialysis

EVD

external ventricular drainage

CT

computerized tomography

WFNS

World Federation of Neurosurgical Societies

GOS

Glasgow outcome scale

NPX

normalized protein expression

LOD

limit of detection

TNF

tumor necrosis factor

TBI

traumatic brain injury

\section{Declarations}

Ethics approval and consent to participate

The Uppsala University Ethical review board for research on humans granted permission for the study. Informed consent for participation was obtained from the patients when feasible or their next of kin.

Consent for publication

Not applicable.

Availability of data and material

The datasets used in the current study are available from the corresponding author on reasonable request.

Competing interests

None.

\section{Funding}

This research project was funded by ALF means (Avtal om läkarutbildning och forskning), that is governmental funding for medical doctors under training for parallel research. 
The first and last author were responsible for the study design and data collection and analysis. The first author was responsible for writing the manuscript. All the other authors contributed with critically revising and improving the manuscript.

\section{Acknowledgements}

The authors would like to thank all the nursing staff at the NICU at Uppsala University Hospital, Uppsala, Sweden for their collaboration in the collection of the CSF samples.

Authors' information (optional)

\section{References}

1. Macdonald RL, Schweizer TA: Spontaneous subarachnoid haemorrhage. Lancet 2017, 389:655-666.

2. Budohoski KP, Guilfoyle M, Helmy A, Huuskonen T, Czosnyka M, Kirollos R, Menon DK, Pickard JD, Kirkpatrick PJ: The pathophysiology and treatment of delayed cerebral ischaemia following subarachnoid haemorrhage. J Neurol Neurosurg Psychiatry 2014, 85:1343-1353.

3. Ohkuma H, Manabe H, Tanaka M, Suzuki S: Impact of cerebral microcirculatory changes on cerebral blood flow during cerebral vasospasm after aneurysmal subarachnoid hemorrhage. Stroke 2000, 31:1621-1627.

4. Alaraj A, Charbel FT, Amin-Hanjani S: Peri-operative measures for treatment and prevention of cerebral vasospasm following subarachnoid hemorrhage. Neurol Res 2009, 31:651-659.

5. Macdonald RL, Kassell NF, Mayer S, Ruefenacht D, Schmiedek P, Weidauer S, Frey A, Roux S, Pasqualin A: Clazosentan to overcome neurological ischemia and infarction occurring after subarachnoid hemorrhage (CONSCIOUS-1): randomized, double-blind, placebo-controlled phase 2 dose-finding trial. Stroke 2008, 39:3015-3021.

6. Macdonald RL, Higashida RT, Keller E, Mayer SA, Molyneux A, Raabe A, Vajkoczy P, Wanke I, Bach D, Frey $A$, et al: Clazosentan, an endothelin receptor antagonist, in patients with aneurysmal subarachnoid haemorrhage undergoing surgical clipping: a randomised, double-blind, placebocontrolled phase 3 trial (CONSCIOUS-2). Lancet Neurol 2011, 10:618-625.

7. Schneider UC, Xu R, Vajkoczy P: Inflammatory events following subarachnoid hemorrhage (SAH). Curr Neuropharmacol 2018.

8. Pluta RM, Hansen-Schwartz J, Dreier J, Vajkoczy P, Macdonald RL, Nishizawa S, Kasuya H, Wellman G, Keller E, Zauner A, et al: Cerebral vasospasm following subarachnoid hemorrhage: time for a new world of thought. Neurol Res 2009, 31:151-158.

9. Sehba FA, Pluta RM, Zhang JH: Metamorphosis of subarachnoid hemorrhage research: from delayed vasospasm to early brain injury. Mol Neurobio/ 2011, 43:27-40. 
10. Macdonald RL: Delayed neurological deterioration after subarachnoid haemorrhage. Nat Rev Neurol 2014, 10:44-58.

11. Lucke-Wold BP, Logsdon AF, Manoranjan B, Turner RC, McConnell E, Vates GE, Huber JD, Rosen CL, Simard JM: Aneurysmal Subarachnoid Hemorrhage and Neuroinflammation: A Comprehensive Review. Int J Mol Sci 2016, 17:497.

12. Provencio JJ: Inflammation in subarachnoid hemorrhage and delayed deterioration associated with vasospasm: a review. Acta Neurochir Supp/ 2013, 115:233-238.

13. de Oliveira Manoel AL, Macdonald RL: Neuroinflammation as a Target for Intervention in Subarachnoid Hemorrhage. Front Neurol 2018, 9:292.

14. Mathiesen T, Edner G, Ulfarsson E, Andersson B: Cerebrospinal fluid interleukin-1 receptor antagonist and tumor necrosis factor-alpha following subarachnoid hemorrhage. J Neurosurg 1997, 87:215220.

15. Fassbender K, Hodapp B, Rossol S, Bertsch T, Schmeck J, Schutt S, Fritzinger M, Horn P, Vajkoczy P, Kreisel S, et al: Inflammatory cytokines in subarachnoid haemorrhage: association with abnormal blood flow velocities in basal cerebral arteries. J Neurol Neurosurg Psychiatry 2001, 70:534-537.

16. Schoch B, Regel JP, Wichert M, Gasser T, Volbracht L, Stolke D: Analysis of intrathecal interleukin-6 as a potential predictive factor for vasospasm in subarachnoid hemorrhage. Neurosurgery 2007, 60:828-836; discussion 828-836.

17. Sarrafzadeh A, Schlenk F, Gericke C, Vajkoczy P: Relevance of cerebral interleukin- 6 after aneurysmal subarachnoid hemorrhage. Neurocrit Care 2010, 13:339-346.

18. Helbok R, Schiefecker AJ, Beer R, Dietmann A, Antunes AP, Sohm F, Fischer M, Hackl WO, Rhomberg P, Lackner P, et al: Early brain injury after aneurysmal subarachnoid hemorrhage: a multimodal neuromonitoring study. Crit Care 2015, 19:75.

19. Niwa A, Osuka K, Nakura T, Matsuo N, Watabe T, Takayasu M: Interleukin-6, MCP-1, IP-10, and MIG are sequentially expressed in cerebrospinal fluid after subarachnoid hemorrhage. $J$ Neuroinflammation 2016, 13:217.

20. Lv SY, Wu Q, Liu JP, Shao J, Wen LL, Xue J, Zhang XS, Zhang QR, Zhang X: Levels of Interleukin1beta, Interleukin-18, and Tumor Necrosis Factor-alpha in Cerebrospinal Fluid of Aneurysmal Subarachnoid Hemorrhage Patients May Be Predictors of Early Brain Injury and Clinical Prognosis. World Neurosurg 2018, 111:e362-e373.

21. Wang Y, Zhong M, Tan XX, Yang YJ, Chen WJ, Liu W, Zheng K: Expression change of interleukin-8 gene in rabbit basilar artery after subarachnoid hemorrhage. Neurosci Bull 2007, 23:151-155.

22. Hanafy KA: The role of microglia and the TLR4 pathway in neuronal apoptosis and vasospasm after subarachnoid hemorrhage. J Neuroinflammation 2013, 10:83.

23. Fisher CM, Kistler JP, Davis JM: Relation of cerebral vasospasm to subarachnoid hemorrhage visualized by computerized tomographic scanning. Neurosurgery 1980, 6:1-9.

24. Hunt WE, Hess RM: Surgical risk as related to time of intervention in the repair of intracranial aneurysms. J Neurosurg 1968, 28:14-20. 
25. Teasdale GM, Drake CG, Hunt W, Kassell N, Sano K, Pertuiset B, De Villiers JC: A universal subarachnoid hemorrhage scale: report of a committee of the World Federation of Neurosurgical Societies. J Neurol Neurosurg Psychiatry 1988, 51:1457.

26. Johansson M, Cesarini KG, Contant CF, Persson L, Enblad P: Changes in intervention and outcome in elderly patients with subarachnoid hemorrhage. Stroke 2001, 32:2845-2949.

27. Jennett B, Bond M: Assessment of outcome after severe brain damage. Lancet 1975, 1:480-484.

28. Assarsson E, Lundberg M, Holmquist G, Bjorkesten J, Thorsen SB, Ekman D, Eriksson A, Rennel Dickens E, Ohlsson S, Edfeldt G, et al: Homogenous 96-plex PEA immunoassay exhibiting high sensitivity, specificity, and excellent scalability. PLoS One 2014, 9:e95192.

29. Dyhrfort P, Shen Q, Clausen F, Thulin M, Enblad P, Kamali-Moghaddam M, Hillered L, Lewen A: Monitoring of Protein Biomarkers of Inflammation in Human Traumatic Brain Injury Using Microdialysis and Proximity Extension Assay Technology in Neurointensive Care. J Neurotrauma 2019.

30. Ericson H, Abu Hamdeh S, Freyhult E, Stiger F, Backryd E, Svenningsson A, Gordh T, Kultima K: Cerebrospinal fluid biomarkers of inflammation in trigeminal neuralgia patients operated with microvascular decompression. Pain 2019, 160:2603-2611.

31. Backryd E, Lind AL, Thulin M, Larsson A, Gerdle B, Gordh T: High levels of cerebrospinal fluid chemokines point to the presence of neuroinflammation in peripheral neuropathic pain: a crosssectional study of 2 cohorts of patients compared with healthy controls. Pain 2017, 158:2487-2495.

32. Zeiler FA, Thelin EP, Czosnyka M, Hutchinson PJ, Menon DK, Helmy A: Cerebrospinal Fluid and Microdialysis Cytokines in Aneurysmal Subarachnoid Hemorrhage: A Scoping Systematic Review. Front Neurol 2017, 8:379.

33. Hollig A, Remmel D, Stoffel-Wagner B, Schubert GA, Coburn M, Clusmann H: Association of early inflammatory parameters after subarachnoid hemorrhage with functional outcome: A prospective cohort study. Clin Neurol Neurosurg 2015, 138:177-183.

34. Savarraj JPJ, Parsha K, Hergenroeder GW, Zhu L, Bajgur SS, Ahn S, Lee K, Chang T, Kim DH, Liu Y, Choi HA: Systematic model of peripheral inflammation after subarachnoid hemorrhage. Neurology 2017, 88:1535-1545.

35. Zhang W, Khatibi NH, Yamaguchi-Okada M, Yan J, Chen C, Hu Q, Meng H, Han H, Liu S, Zhou C: Mammalian target of rapamycin (mTOR) inhibition reduces cerebral vasospasm following a subarachnoid hemorrhage injury in canines. Exp Neurol 2012, 233:799-806.

36. Kubo Y, Koji T, Yoshida J, Ogawa A, Ogasawara K: Predicting neurological deficit severity due to subarachnoid haemorrhage: soluble CD40 ligand and platelet-derived growth factor-BB. Crit Care Resusc 2016, 18:242-246.

37. Hollig A, Stoffel-Wagner B, Clusmann H, Veldeman M, Schubert GA, Coburn M: Time Courses of Inflammatory Markers after Aneurysmal Subarachnoid Hemorrhage and Their Possible Relevance for Future Studies. Front Neurol 2017, 8:694. 
38. Vlachogiannis P, Hillered L, Khalil F, Enblad P, Ronne-Engstrom E: Interleukin-6 Levels in Cerebrospinal Fluid and Plasma in Patients with Severe Spontaneous Subarachnoid Hemorrhage. World Neurosurg 2019, 122:e612-e618.

39. Chen XD, Sun J, Lu C, Ba HJ, Chen MH, Lin JH, Cai JY: The prognostic value of plasma soluble CD40 ligand levels following aneurysmal subarachnoid hemorrhage. Thromb Res 2015, 136:24-29.

40. Kiiski H, Jalkanen V, Ala-Peijari M, Hamalainen M, Moilanen E, Peltola J, Tenhunen J: Plasma Soluble Urokinase-Type Plasminogen Activator Receptor Is Not Associated with Neurological Outcome in Patients with Aneurysmal Subarachnoid Hemorrhage. Front Neurol 2017, 8:144.

41. Gadani SP, Walsh JT, Lukens JR, Kipnis J: Dealing with Danger in the CNS: The Response of the Immune System to Injury. Neuron 2015, 87:47-62.

\section{Figures}

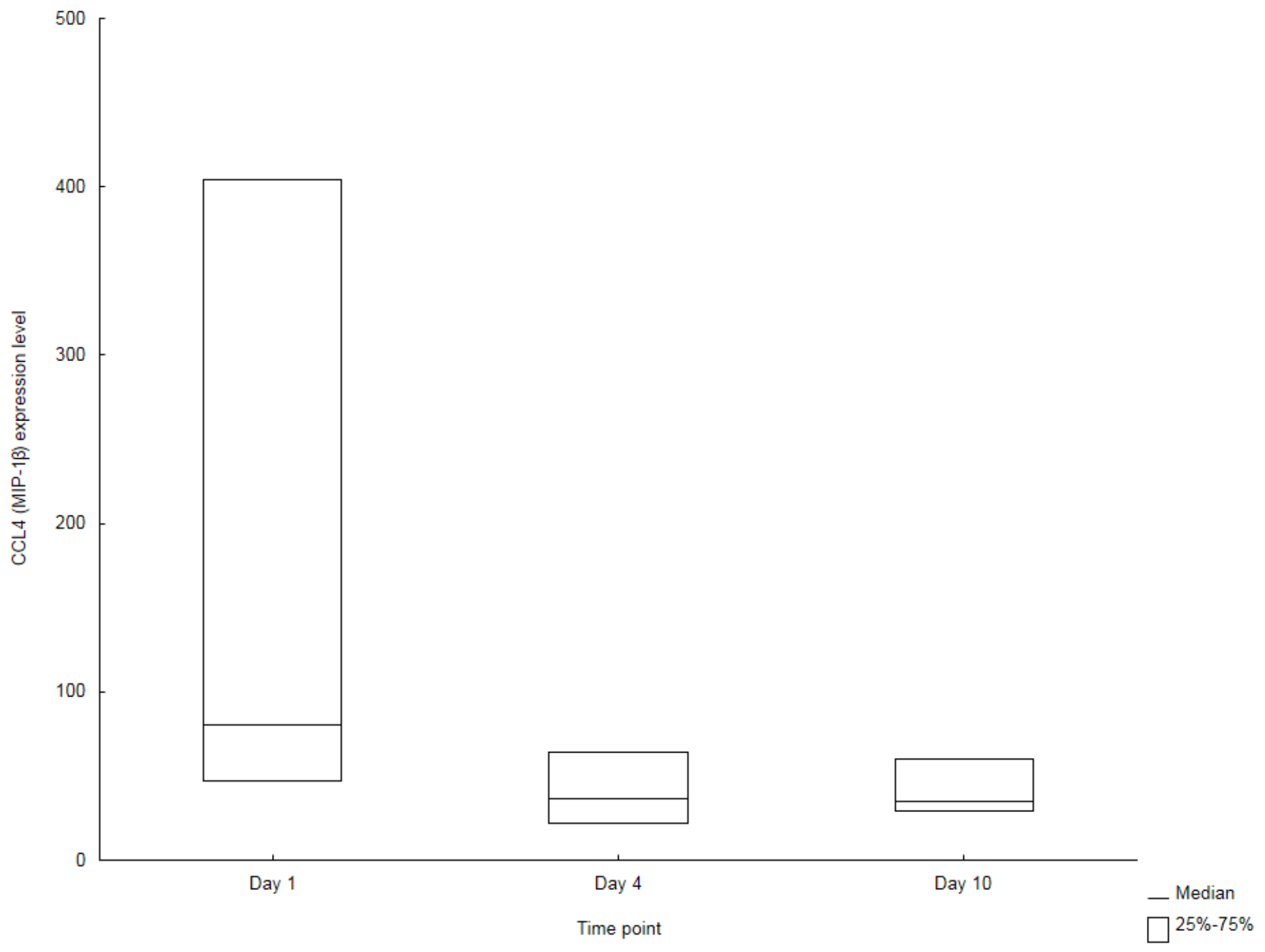

Figure 1 
Group A example; CC4 (or MIP-1ß): temporal pattern of protein expression level (linear NPX) for C-C motif chemokine ligand 4 (CCL4) or macrophage inflammatory protein-1ß (MIP-1ß). Statistically significant early peak (median day 1 vs day 4 values $=80.3$ vs $36.4, p<0.01$ ) but no significantly decreasing trend since values seemed to stagnate between day 4 and 10 (36.4 vs $35.1, p=0.44)$. Of note is the large spread of day 1 values compared to day 4 and day 10 values that were more concentrated.

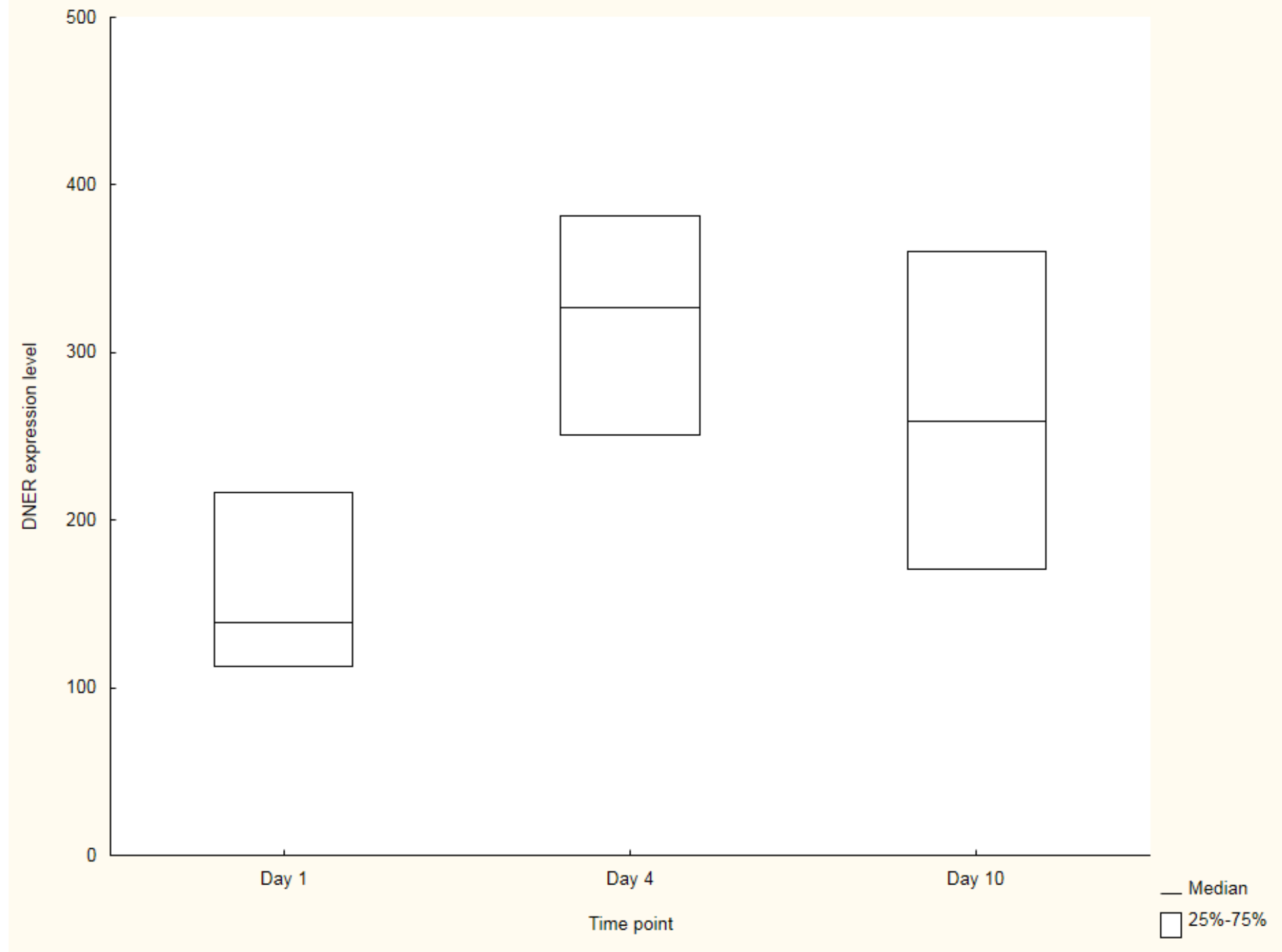

\section{Figure 2}

Group B example; DNER: temporal pattern of protein expression level (linear NPX) for Delta and Notch-like epidermal growth factor-related receptor (DNER). Statistically significant peak in the middle of the observation period defined by significantly higher day 4 vs day $1(327.1$ vs $138.8, p<0.01)$ and day 4 vs day 10 median values (327.1 vs $259.2, p=0.01$ ). 


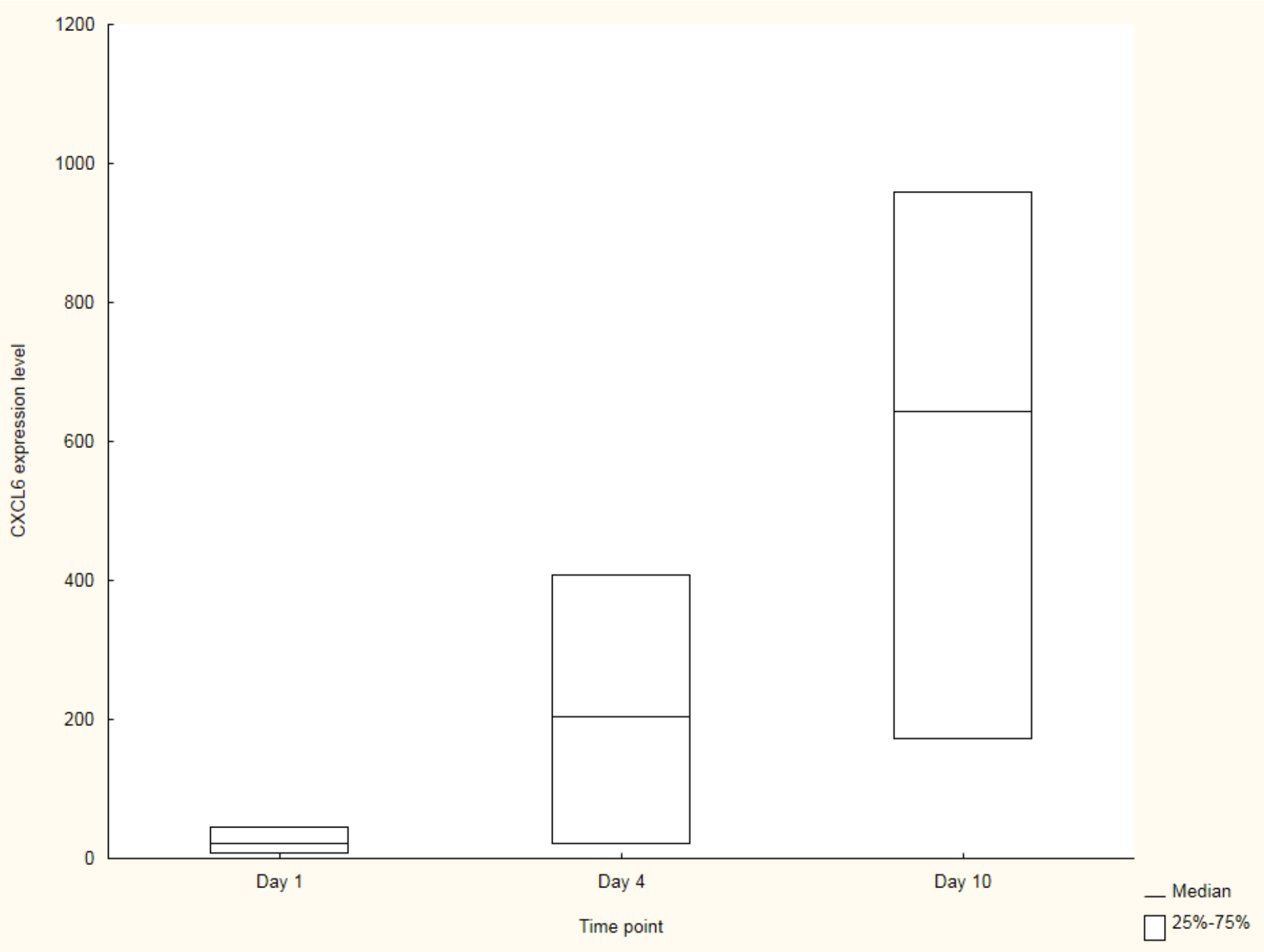

\section{Figure 3}

Group C example; CXCL6: temporal pattern of protein expression level (linear NPX) for C-X-C motif chemokine ligand 6 (CXCL6) showing statistically significant late peak and increasing trend, that is day 1 $<$ day $4<$ day 10 median values ( 21.1 vs 204.3 vs 642.6 respectively, $p<0.01)$. Of note the gradually increasing spread of values towards the end of the observation period. 


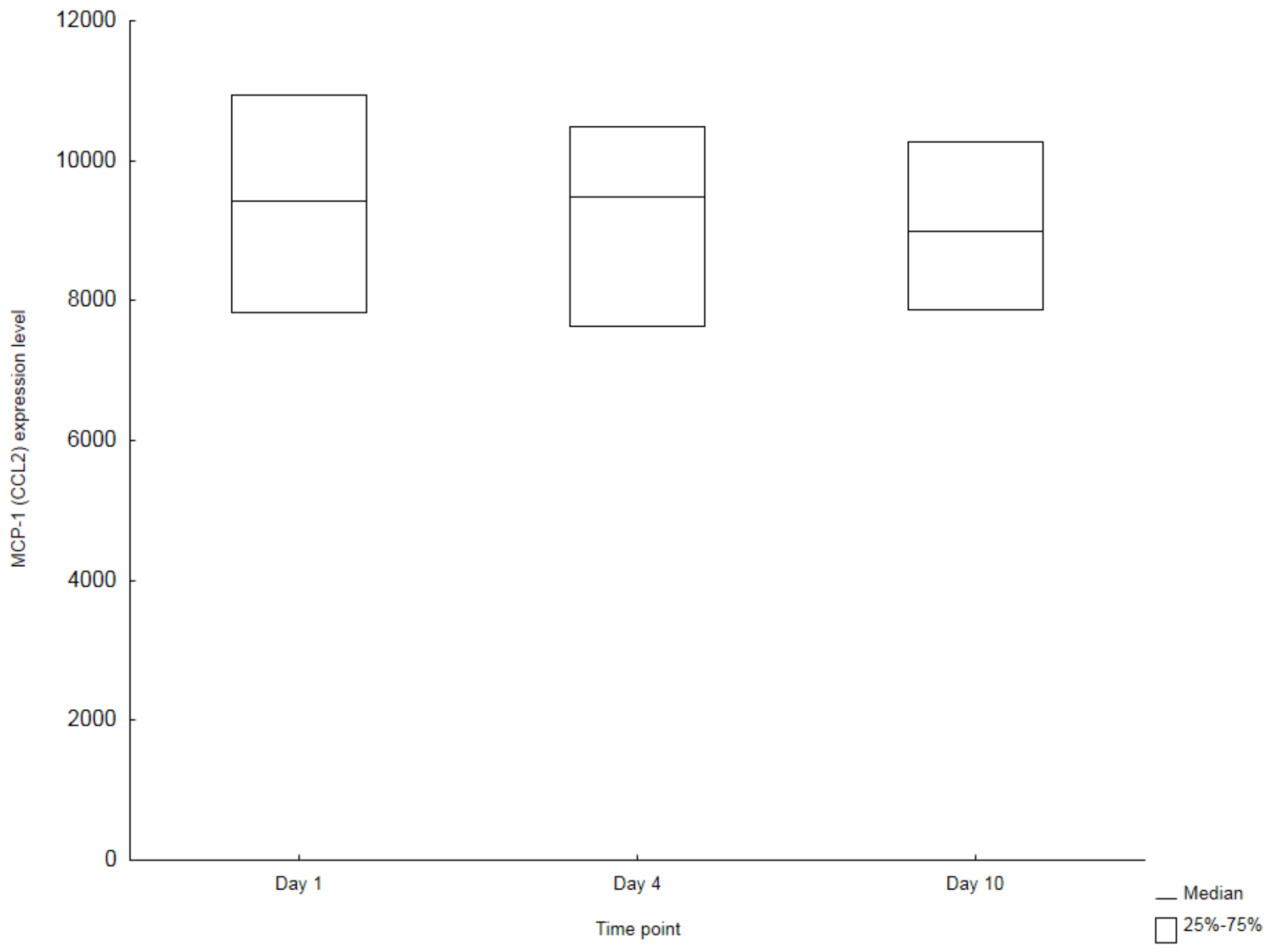

\section{Figure 4}

Group D example; MCP-1: temporal pattern of protein expression level (linear NPX) for monocyte chemotactic protein 1 (MCP-1) or C-C motif chemokine ligand 2 (CCL2) showing essentially stable median values throughout the whole observation period ( 9428.7 vs 9491.3 vs $8998.3, p=0.50$ and $p=0.85$ respectively). 